\title{
Can Vascularity Determine Degenerative Behavior of Uterine Fibroids? Huge Parasitic Uterine Fibroid Mass Mimicking an Ovarian Tumor
}

\author{
John Osaigbovoh Imaralu ${ }^{1}$, Ajani Mustapha ${ }^{2}$, Adebola Yusuf ${ }^{3}, K_{\text {Kolawole Aramide }}^{2}$ \\ Departments of ${ }^{1}$ Obstetrics and Gynaecology, ${ }^{2}$ Histopathology and ${ }^{3}$ Radio-diagnosis, Babcock University Teaching Hospital, \\ Ilishan-Remo, Nigeria.
}

\section{Corresponding Author: \\ Dr. John Osaigbovoh Imaralu}

Email:imaraluj@gmail.com

This is an Open Access article distributed under the terms of the Creative Commons Attribution License (creativecommons.org/ licenses/by/3.0).

Received Accepted

March 27, 2017

Published

June 17, 2017

July 20, 2017

\begin{abstract}
Background: Uterine fibroids have been misdiagnosed for a range of conditions from ovarian to colonic or even retroperitoneal tumors. Apart from the effects of a known and diagnosed fibroid, misdiagnosed fibroids have their own implications for the patient which may include iatrogenic impaired reproductive capability and quality of life. An understanding of fibroid degenerations, especially atypical variants produced by associations between fibroid tumors and bowel or mesentery may help overcome this diagnostic dilemma and aid optimal care. Case Report: The authors report the case of a 30 year old nullipara, who had a huge cystic abdominal tumor with rich vascularity from a broad mesentery, causing complex degenerations and consequently an unusual anatomy. This fibroid tumor was misdiagnosed clinically and at radio-diagnosis for an ovarian malignancy, because of the nature of degenerations that had taken place. Conclusion: An understanding of such atypical degenerations may help to overcome diagnostic dilemma and aid optimal care.
\end{abstract}

Keywords: Humans, Leiomyoma, Mesentery, Ovarian Neoplasms, Uterine Neoplasms.

\section{Introduction}

Leiomyoma or fibroids are benign tumors of the smooth muscles of the uterus and are the most common solid tumors in women worldwide [1]. They account for 70 to $80 \%$ of all new growths in the female genital tract and are the commonest indication for hysterectomy [2]. Although the etiology is poorly understood, its' development is thought to be hormone-mediated as fibroids are known to have more estrogen receptors than normal uterine muscle and the majority atrophy after menopause [3]. Other associations include race, it is commoner in Blacks than in Caucasians [1]. This may be due to higher prevalence of pelvic infections in the Black race with reactive abnormal uterine growth. This higher prevalence in blacks is also thought to result from the presence of a gene encoding for fibroid [4]. While it is rare before the age of 20 years, it is commoner in nulliparous and relatively infertile women $[1,4]$.

Leiomyoma have a range of effect depending on the location within and outside the uterus, the hormonal status of the woman (e.g pregnant or menopausal), the size of the fibroid and the nature of the degenerations that are present within it. Uterine fibroids have been misdiagnosed for a range of conditions from ovarian tumors to colonic or even retroperitoneal tumors. Apart from the effects of a known and diagnosed fibroid, misdiagnosed fibroids have their own implications for the patient and her management.

A recognized cause of fibroid misdiagnosis is the nature of fibroid degenerations. Uterine fibroids may be confused with malignant abdominal or pelvic tumors, especially when they are adherent to or receive vascular supply from adjacent bowel 
or mesentery [5]. We present here, the case of a 30 year old nullipara in whom a uterine fibroid tumor was observed to have a rich vascular trunk from the omentum at laparotomy with possible resultant multiple complex degenerations.

\section{Case Report}

A 30 year old, single, nullipara female presented with two year history of progressive abdominal swelling. Although she noticed that the mass had increased rapidly in size in the preceding year, with associated early satiety, there was no pain, abnormal vaginal bleeding, heavy menstrual bleeding, weight loss, or other gastrointestinal or genitourinary symptoms.

She attained menarche at 11 years and menstruated for five days in a regular 28 day cycle. She was not on any form of contraceptives, or hormonal therapy. She had no chronic medical illness and was not on any routine medication. She had never used herbal concoctions or other drugs for tumor shrinking and had never undergone any surgery in the past. She was a secondary school leaver, single and a trader. Examination revealed a young woman who was not in any distress and her BMI was $38.9 \mathrm{~kg} / \mathrm{m}^{2}$. Her abdomen was distended with a 34 week size pelvic mass, which was firm, non-tender, with well-defined smooth edges. There was no demonstrable ascites and rectal examination revealed, a freely mobile rectal mucosa, devoid of masses or evidence of bleeding.

An assessment of a huge abdomino-pelvic mass was made to rule out ovarian tumor. Her investigation results were as follows: packed cell volume $=34.7 \%$, total leukocyte count $=7.5 \times 10^{6} / \mathrm{L}$, platelet count $=185,000 / \mathrm{mm}^{3} \mathrm{CA}-125=51.36 \mathrm{U} / \mathrm{mL}$ $(0-35 \mathrm{U} / \mathrm{mL})$, and CEA $=1.14 \mathrm{ng} / \mathrm{mL}(0-5.0 \mathrm{ng} / \mathrm{mL})$. Kidney and liver function test results were all normal. Abdomino-pelvic ultrasound scanning revealed a 'huge right ovarian cystic mass with solid components, but no septa'. Contrast enhanced Computed Tomography (CT) scan also had findings suggestive of an ovarian tumor [Fig.1]. Intravenous

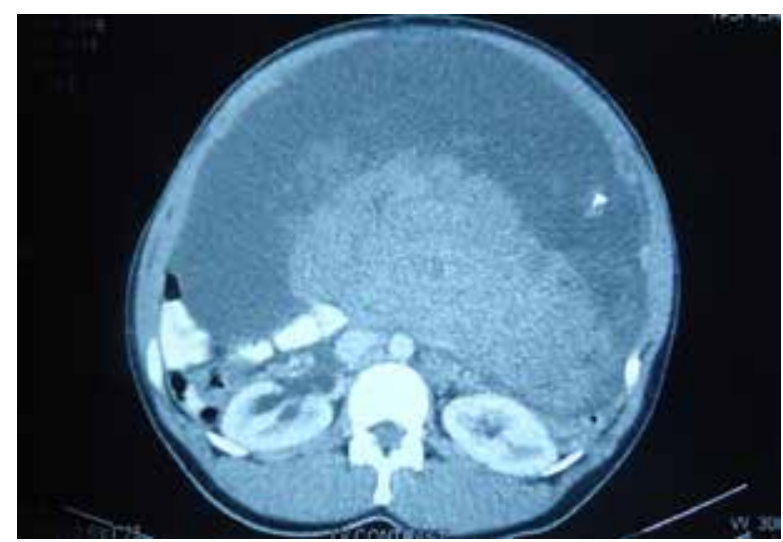

Fig.1: CT scan image of the abdomen-pelvic mass showing the areas of degeneration.

urogram revealed mild left hydroureter with lateral deviation in the distal part.

Exploratory laparotomy showed ascites, huge sub-serous saucer-shaped uterine fundal mass with concavity towards the uterus. It had a widest transverse diameter of $50 \mathrm{~cm}$ and a thickness of 25 $\mathrm{cm}$. The mass had a thick white capsule with a large straw-colored fluid-filled cavity and interspersed hard and soft masses palpable through the lower aspect adjoining the stalk. The mass was attached at its center to the fundus of the uterus by an $8 \mathrm{~cm}$ long and $6 \mathrm{~cm}$ wide stalk. Upon removal from the uterus the entire mass weighed $11 \mathrm{~kg}$, the cystic part ruptured however soon after weighing resulting in release of the fluid contents. There was also a $15 \mathrm{~cm}$ wide omental fold on the convex superior surface of the mass which contained a rich network of several large dilated blood vessels measuring upto $5 \mathrm{~mm}$ in diameter, reaching and exiting the mass.

The uterus was enlarged and tubular in shape, it also contained fibroid nodules of varying sizes ranging from $2 \mathrm{~mm}$ to $10 \mathrm{~mm}$ diameter in the anterior and posterior myometrium [Fig.2]. Both ovaries were visualized and noted to be grossly healthy looking, with a stigma of recent ovulation on the left. The fallopian tubes, the adjoining loops of bowel, urinary bladder, the inferior aspect of the liver and the pouch of Douglas all looked grossly 
healthy and had no tumor or endometriotic deposits. There were no enlarged lymph nodes in the paraaortic, external iliac, inguinal or para-rectal areas. An excisional biopsy was done involving the removal of the entire fibroid tumor and stalk via a wedge shaped incision on the uterus. This was preceded by an initial omental resection to free the superior attachment and obtain specimen for histology. Fundal metroplasty was then done after removing fibroids in the body of the uterus.

She had an uneventful post-operative recovery and was discharged home after seven days. She was seen at the gynaecology clinic and reviewed with the histo-pathology report which revealed macroscopically - a huge encapsulated mass weighing 6000 grams and measuring $21 \times 20 \times 13 \mathrm{~cm}$. Cut section showed greyish white appearance arranged in whorled pattern. There were also areas of calcification and cystic changes within the tumor. Microscopic sections revealed a benign mesenchymal neoplasm composed of interlacing bundles of smooth muscle fibres few of which were disposed in whorled pattern. There were also areas of calcification and hyalinization within the neoplasm [Fig.3,4].

\section{Discussion}

The clinical presentations and complications of uterine fibroids are varied, ranging from menorrhagia, abdominal swelling, abdominal pain, dysmenorrhea to infertility, miscarriage and preterm delivery. Apart from the site, size, number and location of the leiomyoma, degenerative changes are major modulators of the clinical presentations and complications of uterine fibroids [1]. The above mentioned patient presented with progressively increasing abdomino-pelvic mass which is a nonspecific feature that can be due to other pathology including an ovarian tumor. A clinical diagnosis of an ovarian tumor was made because ovarian neoplasms have a tendency to behave in such nonspecific manner and grow to such huge size, with minimal symptoms. The absence of menorrhagia

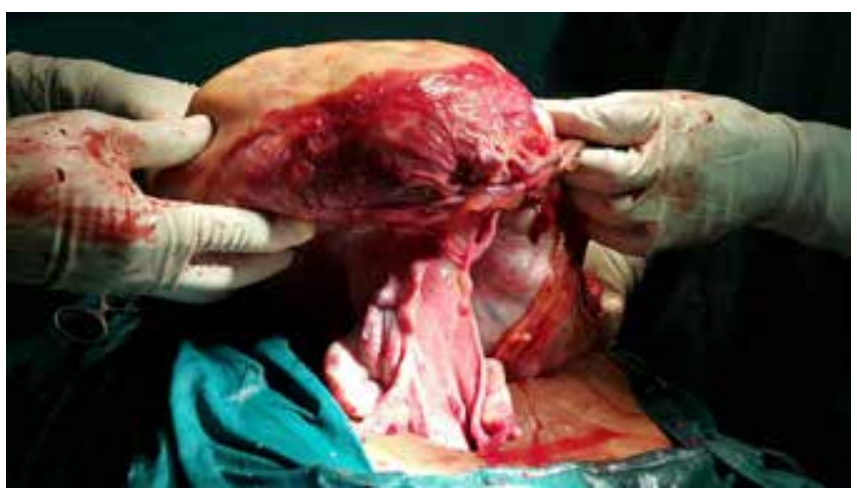

Fig.2: Huge degenerated fibroid with the extensive blood supply running within mesentery. Note that the ovaries and the fallopian tubes are present.

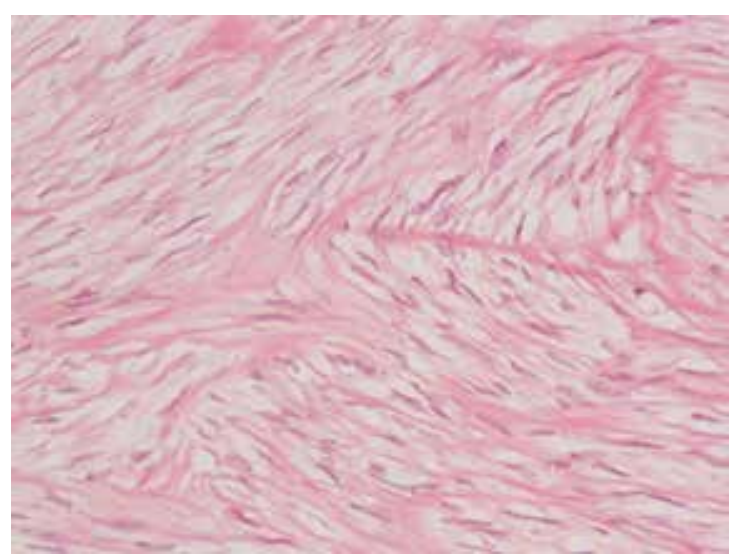

Fig.3: Proliferating smooth muscle cells (arrow) disposed in interlacing fascicles and whorls consistent with leiomyoma (Haematoxylin and Eosin, ×400).

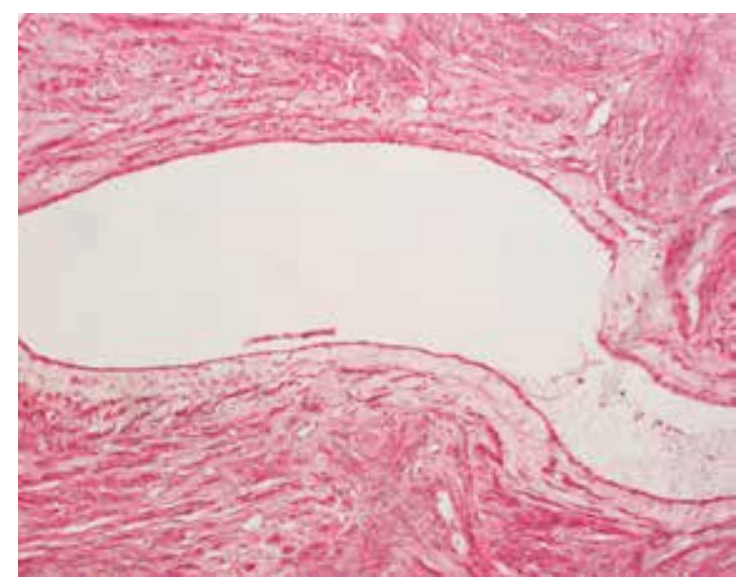

Fig.4: Proliferating smooth muscle cells disposed in interlacing fascicles and whorls with focal area of cystic degeneration (arrow) consistent with leiomyoma (Haematoxylin and Eosin, $\times 100$ ). 
and other menstrual disorders, the huge size of the tumor and its cystic consistency on examination; made an ovarian tumor, a more likely diagnosis.

The findings from ultrasound scanning largely supported ovarian tumor; the size and the ultrasonic components of the mass, cystic and calcific solid components led to the misdiagnosis. The findings from contrast-enhanced CT- scanning were essentially in support of an ovarian tumor. The absence of suggestive history and examination, non-suggestive tumor markers failed to suggest malignant process. These tumor markers are not specific to ovarian tumors [6]. The major cause of the diagnostic dilemma in this case was the nature of the degenerations which affected the anatomy of the mass. Degenerations have been reported to be prevalent in $5 \%$ of patients undergoing uterine fibroid embolization therapy [7]. The well documented types of fibroid degenerations are hyaline, cystic, red, fatty and calcific degeneration [2]. The major reason for fibroid degenerations is thought to be relative ischemia as the leiomyoma out-grows its supply from the nearby blood vessels with depletion of oxygen and nutrients [8]. This theory pre-supposes that degenerations can occur only in large leiomyoma's. A study involving the use of MRI however, reported degenerations in fibroids as small as $5-8 \mathrm{~cm}$ in diameter [7] sizes that are not usually associated with ischemia suggesting the possible existence of another mechanism of occurrence of degenerations in uterine fibroids. Hyaline degeneration is the commonest type of degeneration accounting for approximately $60 \%$ of all fibroid degenerations $[8,9]$. Cystic degeneration occurs in approximately $4 \%$ of leiomyoma and typically follows hyaline degeneration [9]. Although this hyaline-cystic transformation may be applied as the mechanism for the nature of the fibroid degeneration found in our patient. The fibroid mass was however noted to have a rich network of omental blood supply suggesting a role for this rich vascularity in causing a de-novo cystic component of the tumor rather than the ischemia- hyaline-cystic model. A case was reported of uterine fibroids being misdiagnosed for GastroIntestinal Stromal Tumor (GIST), examination of that tumor revealed that the sub-serosal fibroids like those found in our patient were adherent to the bowels and mesentery [10].

Red degeneration is due to hemorrhagic infarction of the uterine leiomyoma, which is a recognized complication, especially during pregnancy. Venous obstruction at the periphery of the lesion has been suggested as the mechanism of red degeneration. This is thought to induce hemorrhagic infarction and extensive necrosis involving the whole fibroid tumor [11]. Red degeneration is found in about $3 \%$ of all uterine leiomyoma and in $8 \%$ of fibroid tumors in pregnancy [11]. As high as $42 \%$ of patients with red degeneration were reported in a study to have been pregnant within the preceding two years of diagnosis of the fibroid degeneration [7]. Red degeneration was not found on examination of fibroids removed from our patient who was a nulligravida.

Surgical options include myomectomy and hysterectomy. Myomectomy is preferred in symptomatic young women with future reproductive desire while hysterectomy is the treatment of choice in older women who have completed their family and in cases where myomectomy is deemed to be exceedingly more hazardous than hysterectomy. Hysterectomy however remains the only curative treatment for fibroids $[3,12]$.

The surgery in this patient did not include hysterectomy or oophorectomy because the ovaries and the other parts of the uterus below the tumor stalk looked grossly healthy, there was no ascites or peritoneal seedling of any kind, more so our patient was 30 years old and nulliparous, she did not have any risk factor that was specific for leiomyosarcoma like use of tamoxifen, exposure to irradiation, retinoblastoma, renal cell carcinoma and other childhood tumors or exposure to antineoplastic drugs [13]. 
Misdiagnosis of degenerated leiomyoma has been reported to result in unnecessary multiorgan excisions [10], since the histopathology reports usually just like in our case came out to be negative for any evidence of malignancy. It has been suggested that an excisional biopsy of the pelvic mass be done when a degenerated leiomyoma is thought to mimic a pelvic malignancy, the histology report should be reviewed and definitive therapy scheduled without delay thereafter [10]. The major reason for this note of caution is the fact that leiomyosarcomas are rare especially in young women. A systematic review reported prevalence rates ranging from 0.05 ( 1 in 2000) to $0.28 \%$ ( 1 in 352 ) of leiomyosarcoma in specimen obtained from surgeries for uterine fibroid [14]. Another similar study reported, that the risk of occult leiomyosarcoma in myomectomy specimen obtained from patients aged $<40$ years was 1 in 2337 [15].

The main limitation of our report is the inability to fully establish the link (by growth monitoring) between mesenteric vasculature and the nature of the fibroid degeneration, a prospective cohort study involving MRI follow up of small asymptomatic fibroid nodules would be useful to solve this puzzle.

\section{Conclusion}

Fibroid misdiagnosis may result in treatment that may impair reproductive capability and quality of life. An understanding of fibroid degenerations, especially atypical degenerations caused by associations between fibroid tumors and bowel or mesentery may help overcome this diagnostic dilemma and aid optimal care.

Contributors: JOI assessed the patient clinically and performed the surgery with his surgical team, he also wrote the initial draft of the manuscript with substantial contribution from AM. AM and KA performed the histologic diagnosis while AY performed the radiologic diagnosis. JOI will act as guarantor. All authors read and approved the final draft of the manuscript and certified it for publication.

Funding: None; Competing interests: None stated.

\section{References}

1. Lumsden MA. Benign disease of the uterus. In: Edmonds DK (ed), Dewhurst's textbook of Obstetrics and Gynaecology for Postgraduates, $7^{\text {th }}$ edition. Blackwell Scientific Publication, Oxford. 2007:638-644.

2. Akinyemi BO, Adewoye BR, Fakoya TA. Uterine fibroid: a review. Nig J Med. 2004:13:318-329.

3. Chalas E, Constantino JP, Wickerham DL, Wolmark $\mathrm{N}$, Lewis GC, Bergman $\mathrm{C}$, et al. Benign gynecologic conditions among participants in the Breast Cancer Prevention Trial. Am J Obstet Gynecol. 2005;192:12301237.

4. Okezie O, Ezegwui HU. Management of uterine fibroids in Enugu, Nigeria. J Obstet Gynaecol. 2006;26:363-365.

5. Abraham SC. Distinguishing gastrointestinal stromal tumors from their mimics: an update. Advances in Anatomic Pathology. 2007;14:178-188.

6. Hanoch J, McIndoe A. Improving the prognosis of ovarian cancer. In: Studd J (ed), Progress in Obstetrics and Gynaecology, Vol.16, Elsevier, London: 2005; pp. 257-370.

7. Han SC, Kim MD, Jung DC, Lee M, Lee MS, Park S, et al. Degeneration of leiomyoma in patients referred for uterine fibroid embolization: incidence, imaging features and clinical characteristics. Yonsei Med J. 2013;54:215219.

8. Okizuka H, Sugimura K, Takemori M, Obayashi C, Kitao M, Ishida T. MR detection of degenerating uterine leiomyomas. J Comput Assist Tomogr. 1993;17:760766.

9. Ueda H, Togashi K, Konishi I, Kataoka ML, Koyama T, Fujiwara $\mathrm{T}$, et al. Unusual appearances of uterine leiomyomas: MR imaging findings and their histopathologic backgrounds. Radiographics. 1999;19:S131-45.

10. Zhu X, Fei J, Zhang W, Zhou J. Uterine leiomyoma mimicking a gastrointestinal stromal tumor with chronic spontaneous hemorrhage: A case report. Oncology Letters. 2015;9;2481-2484.

11. Kawakami S, Togashi K, Konishi I, Kimura I, Fukuoka M, Mori T, et al. Red degeneration of uterine leiomyoma: MR appearance. J Comput Assist Tomogr. 1994;18:925928.

12. Akinola OI, Fabamwo AO, Akinola RA, Ottun TA, Akinniyi A, Akpan AE. Uterine artery ligation for the treatment of fibroids. Acta Obstet Gynecol Scand. 2009;88:59-62.

13. Hosh M, Antar S, Nazzal A, Antar S, Nazzal A, Warda M, Gibreel A, Refky B. Uterine Sarcoma: Analysis of 13,089 cases based on surveillance, epidemiology, and end results database. Int J Gynecol Cancer. 2016;26:1098. 
14. Pritts EA, Vanness DJ, Berek JS, Parker W, Feinberg R, Feinberg J, et al. The prevalence of occult leiomyosarcoma at surgery for presumed uterine fibroids: a meta-analysis. Gynecol Surg. 2015;12:165.
15. Wright JD, Tergas AI, Cui R, Burke WM, Hou JY, Ananth $\mathrm{CV}$, et al. Use of Electric Power morcellation and prevalence of underlying cancer in women who undergo myomectomy. JAMA Oncol. 2015;1:69. 